\title{
Remodelación de papila gingival interdental con ácido hialurónico. Una solución estética.
}

\section{Remodeling interdental gingival papilla with hyaluronic acid. An aesthetic solution}

\author{
Javiera Iribarra-Leigh ${ }^{1}$, María Soto-Royo ${ }^{1}$, María Rubio-Muñoz ${ }^{1}$, Oscar Torres-Castillo", \\ Loreta Baldeig ${ }^{1}$, Andrés Gómez-Morales ${ }^{1 *}$
}

1. Facultad de Odontología, Universidad del Desarrollo, Concepción, Chile.

* Correspondencia autor: Andrés Gómez-Morales | Dirección. Freire 1799. Concepción. | Teléfono: +56 9 92810492. | E-mail:andresgomez@udd.cl Trabajo recibido el 16/01/2019.

Aprobado para su publicación el 04/08/2019.

\section{RESUMEN}

El objetivo de este trabajo es evaluar el efecto de remodelación y relleno mediante aplicación de ácido hialurónico en papilas gingivales de sector estético con defectos de triángulo negro en un paciente tratado periodontalmente. Se inyectaron $0.15 \mathrm{ml}$ de gel de ácido hialurónico dividido en 3 sesiones. Clínicamente se observó aumento de volumen y aumento de dimensiones verticales y horizontales en las papilas, mejorando así los parámetros estéticos. Como conclusión la aplicación de ácido hialurónico es efectiva en la remodelación y relleno en pérdidas de papila clase I de Nordland y Tarnow.

PALABRAS CLAVE:

Papila interdental; Encía; Sonrisa estética.

Rev. Clin. Periodoncia Implantol. Rehabil. Oral Vol. 12(3); 151-153, 2019.

\section{ABSTRACT}

The objective of this work is to evaluate the effect of remodeling and filling through the application of hyaluronic acid in gingival papilla in the aesthetic sector with black triangle defects, in a periodontally- treated patient. In 3 portioned sessions, $0.15 \mathrm{ml}$ of hyaluronic acid gel was injected. Clinically, an increase in volume was observed, and vertical and horizontal dimensions in the papilla increased too, thus improving aesthetic parameters. In conclusion, the application of hyaluronic acid is effective in the remodeling and filling in losses of class I papilla (Nordland and Tarnow classification).

\section{KEY WORDS:}

Interdental papilla; Aesthetic smile; Gingiva.

Rev. Clin. Periodoncia Implantol. Rehabil. Oral Vol. 12(3); 151-153, 2019.

\section{INTRODUCCIÓN}

La pérdida de altura de la papila interdental es una secuela común de la enfermedad periodontal y su tratamiento(1). La pérdida de papila, con la consiguiente aparición de triángulos negros genera inquietud en los pacientes por las consecuencias estéticas observadas en la región anterior maxilar, sobre todo durante la sonrisa ${ }^{(2)}$. Por otra parte, la pérdida de papila interdental interfiere funcionalmente, ya que una papila normal evita la acumulación de restos de alimentos y evita el escape de saliva o aire cuando hablamos ${ }^{(3)}$. Desde el punto de vista fisiológico, la papila interdental al comportarse como barrera biológica, protege las estructuras periodontales más profundas.

Actualmente se utilizan diversas técnicas para el aumento de altura en papilas interdentales o disminución del efecto del triángulo negro, incluyendo tratamientos quirúrgicos, ortodóncicos, protésicos o combinaciones de estos, sin embargo estos métodos son poco predictivos y altamente invasivos ${ }^{(4)}$. En base a lo anterior, surge como tratamiento alternativo el uso de ácido hialurónico. Si bien este tratamiento ha sido estudiado y documentado, no es de uso habitual en la práctica clínica odontológica.

El ácido hialurónico es miembro de la familia de los glicosaminoglicanos y un componente principal de la matriz extracelular de casi todos los tejidos $^{(4)}$. La mayoría de las células son capaces de producirlo y su función más importante es la participación en la curación y reparación de tejidos ${ }^{(5)}$ Las propiedades del ácido hialurónico, como su biocompatibilidad, hidrofilicidad(4) y su carácter no inmunogénico, además de los efectos antisépticos, antiinflamatorios y bacteriostáticos descritos han permitido su aplicación tópica para la cicatrización de heridas, en tratamiento de gingivitis, periodontitis y por inyección para restaurar el volumen de las papilas interdentales(6). Es importante mencionar que geles de ácido hialurónico inyectables disponibles en la actualidad son polímeros que se formulan mediante reticulación de ácido hialurónico producido por especies de Streptococcus ${ }^{(6)}$.

El objetivo de este trabajo es evaluar el efecto de remodelación y relleno papilar mediante la aplicación de ácido hialurónico en papilas gingivales con defectos de triángulo negro, en el sector estético en un paciente periodontalmente tratado y sano en etapa de mantención periodontal.

\section{MATERIALES Y MÉTODO.}

Paciente sexo femenino de 23 años de edad, sin antecedentes mórbidos relevantes y tratada periodontalmente. Presenta defectos de triángulo negro en papilas gingivales del sector anterior del maxilar superior (Figuras 1 y 2 ).

Las papilas fueron clasificadas según la clasificación de Norland y 
Tarnow ${ }^{(1)}$, dando los siguientes resultados. La papila entre los dientes $1.3-1.2$ clase III, papila entre $1.2-1.1$ clase II (Figura 1), papila entre $1.1-2.1$ clase I, papila entre $2.1-2.2$ clase I (Figura 2 ) y papila entre $2.2-2.3$ clase II.

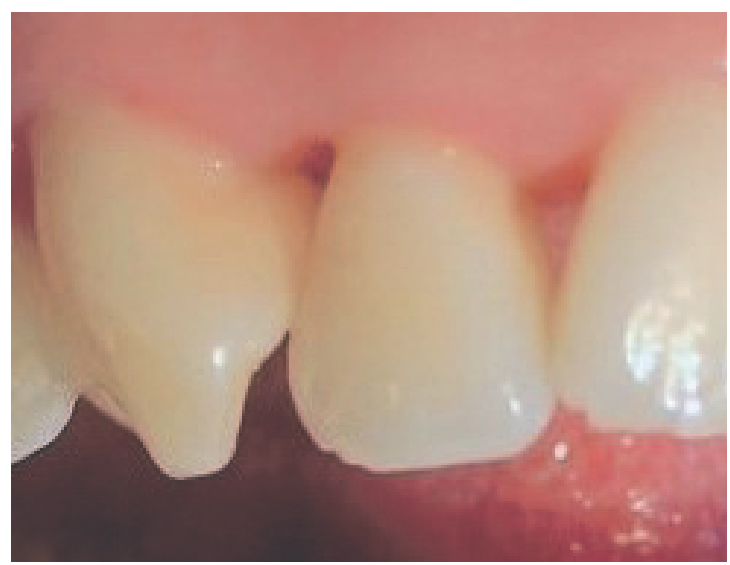

Figura 1. Papila 1. 1-1.2 inicial

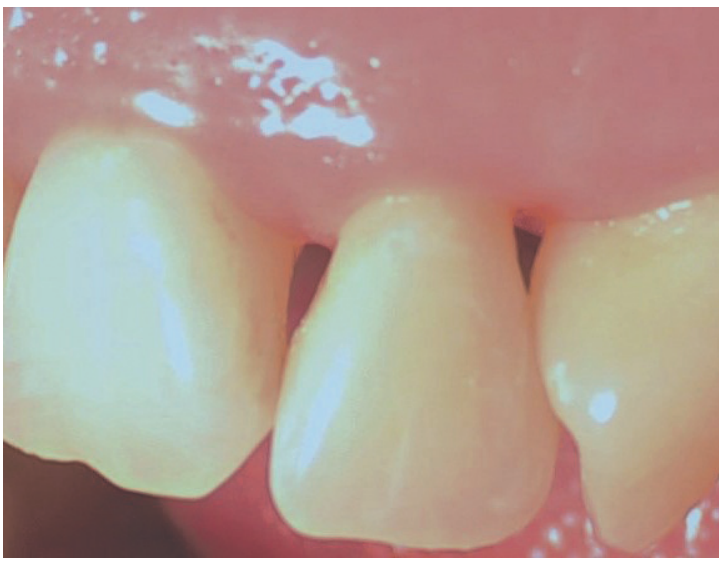

Figura 2. Papila 2.1-2-2 inicial

Además, para evaluar los resultados de forma numérica, se estandarizaron las medidas de cada uno de los defectos de triángulo negro mediante dos sondas periodontales (PCPUNC156, Hu-Friedy ${ }^{\circledR}$ ). La primera sonda se ubicó en sentido horizontal, a modo de tangente sobre los cénit que formaban el defecto de triángulo negro, la segunda sonda se ubicó perpendicular a la primera y a través del eje mayor de la papila para medir el defecto de triángulo negro. También, y con sonda periodontal se midió a través del surco periodontal, la distancia entre la cresta ósea y el punto de contacto para obtener una estimación porcentual de relleno papilar. Este procedimiento se realizó en cada una de las papilas involucradas. (Tabla 1)

Tabla 1. Porcentaje de relleno papilar.

\begin{tabular}{|c|c|c|}
\hline \multicolumn{3}{|c|}{ Porcentaje de relleno papilar } \\
\hline Papilas & $\begin{array}{c}\text { Distancia C.O. }- \\
\text { P.C. }\end{array}$ & \% de cobertura \\
\hline $1.3-1.2$ & $6 \mathrm{~mm}$ & $56 \%$ \\
\hline $1.2-1.1$ & $6 \mathrm{~mm}$ & $56 \%$ \\
\hline $1.1-2.1$ & $5 \mathrm{~mm}$ & $100 \%$ \\
\hline $2.1-2.2$ & $6 \mathrm{~mm}$ & $56 \%$ \\
\hline $2.2-2.3$ & $6 \mathrm{~mm}$ & $56 \%$ \\
\hline
\end{tabular}

C.O: Cresta ósea. P.C: Punto de contacto.

Luego, en cada uno de los defectos se aplicó ácido hialurónico inyectable, Hyalsense fine ${ }^{\circledR}$, de laboratorios Abbott. En total, se inyectaron $0.15 \mathrm{ml}$ de gel de ácido hialurónico reticulado, dividido en tres sesiones con dos semanas de intervalo. Por lo tanto, en cada sesión se inyectó $0.05 \mathrm{ml}$ de gel de ácido hialurónico. La aplicación se realizó 2 a $3 \mathrm{~mm}$ en vestibular, apical a la punta coronal de la papila. Se pidió al paciente que no se cepillara y no usara elementos de higiene interproximal 24 horas post aplicación en la zona intervenida.

\section{RESULTADOS.}

Se evaluó a los 60 días post-aplicación, observándose clínicamente aumento de volumen en sentido vertical y horizontal de las papilas (Figuras 3 y 4 ), mejorías en los parámetros estéticos y ausencia de signos clínicos de inflamación. Se clasificaron los resultados en: triángulo negro cubierto totalmente y triángulo negro cubierto parcialmente. Además se graficó en una tabla la cantidad de milímetros ganados en cada papila. (Tabla 2).

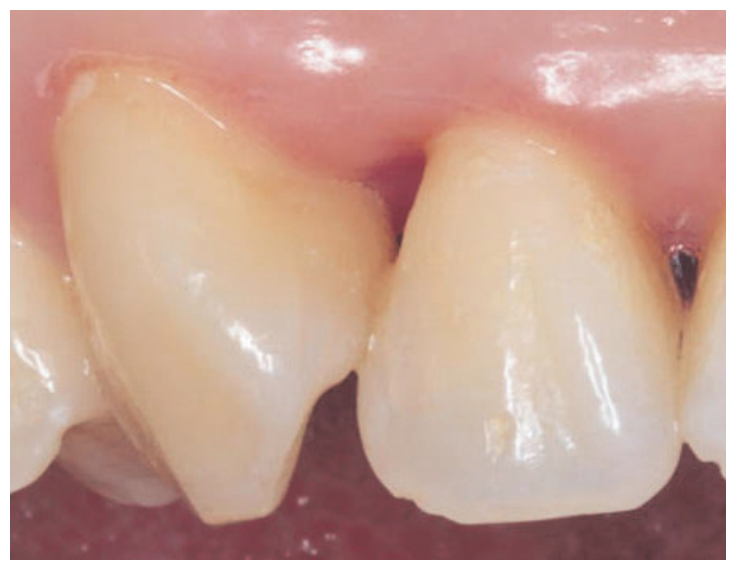

Figura 3. Papila 1.1-1.2 final

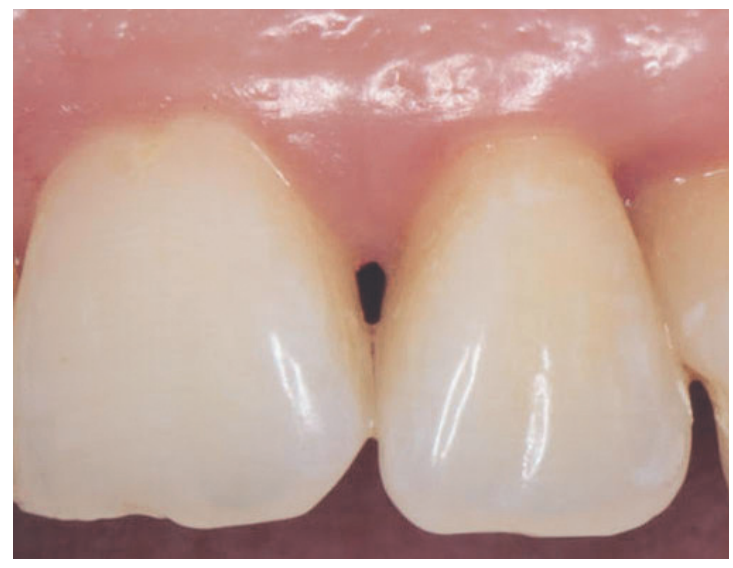

Figura 4. Papila 2.1-2.2 final

Tabla 2. Medidas verticales de la papila $(\mathrm{mm})$

\begin{tabular}{|c|c|c|c|}
\hline \multicolumn{4}{|c}{ Medidas verticales de las papilas } \\
\hline Papilas & $\begin{array}{c}\text { Defecto de } \\
\text { triángulo } \\
\text { negro inicial }\end{array}$ & $\begin{array}{c}\text { Defecto de } \\
\text { triángulo } \\
\text { negro final }\end{array}$ & $\begin{array}{c}\text { Cantidad de } \\
\text { mm ganados } \\
\text { por papila. }\end{array}$ \\
\hline $1.3-1.2$ & $2 \mathrm{~mm}$ & $1 \mathrm{~mm}$ & $1 \mathrm{~mm}$ \\
\hline $1.2-1.1$ & $3 \mathrm{~mm}$ & $1 \mathrm{~mm}$ & $2 \mathrm{~mm}$ \\
\hline $1.1-2.1$ & $2 \mathrm{~mm}$ & $0 \mathrm{~mm}$ & $2 \mathrm{~mm}$ \\
\hline $2.1-2.2$ & $3 \mathrm{~mm}$ & $1 \mathrm{~mm}$ & $2 \mathrm{~mm}$ \\
\hline $2.2-2.3$ & $2 \mathrm{~mm}$ & $1 \mathrm{~mm}$ & $1 \mathrm{~mm}$ \\
\hline
\end{tabular}

\section{DISCUSIÓN}

La finalidad de este caso es poder evaluar un método mínimamente invasivo para aumentar las papilas interdentales. Considerando que los 
derivados del ácido hialurónico están disponibles y proporcionan un aumento seguro y efectivo de tejidos blandos en el enfoque integral de rejuvenecimiento facial no quirúrgico ${ }^{(7)}$. Se han propuesto un sin número de tratamientos para resolver el problema estético de los defectos de triángulo negro, dentro de ellos tenemos el curetaje periódico ${ }^{(9)}$, la técnica de Beagle ${ }^{(10)}$, una combinación de técnica Roll y preservación de la papila(11) y el colgajo pediculado ${ }^{(12)}$. Todas técnicas invasivas. Los resultados de nuestro estudio demostraron que la aplicación de gel de ácido hialurónico fue más exitosa para los defectos de triángulo negro clase I, que para los defectos de triángulo negro clase II y III de Nordland y Tarnow. Estos resultados pueden ser comparados con los obtenidos por Becker et al. ${ }^{(13)}$ y Sadat et al(5).

En el estudio de Becker et al, se evaluaron 14 papilas adyacentes a dientes e implantes y en el $70 \%$ de las muestras se logró una mejora del $94 \%$ a los 25 meses. En el estudio de Sadat et al, se evaluaron 21 papilas, todas adyacentes a dientes, con un seguimiento de 6 meses se observó más del $50 \%$ de mejoría en el $43 \%$ de los casos. En nuestro estudio se evaluaron 5 papilas, todas adyacentes a dientes, con seguimiento de 2 meses, donde en 2 de ellas se obtuvo un $100 \%$ de cobertura y en 3 de ellas un $56 \%$. Cabe mencionar que el estudio de Becker et al. y de Sadat et al. fueron con protocolos de aplicación, muestras y seguimientos distintos. No obstante, el uso de una técnica más segura y menos invasiva, como la aplicación del ácido hialurónico, se puede considerar como una alternativa para e aumento de papila interdental(14)

\section{CONCLUSIÓN}

La aplicación de ácido hialurónico es efectiva en la remodelación y relleno en pérdidas de papila clase I de Nordland y Tarnow intervenidas en este estudio. Por el contrario, no se logró remodelar y rellenar por completo los defectos de triángulo negro clase II y III de Norland y Tarnow. Es necesaria la aplicación periódica de geles de ácido hialurónico, cada 6 meses aproximadamente, para mantener los resultados a través de tiempo.

\section{RELEVANCIA CLÍNICA}

Justificación científica para el estudio está determinada por la búsqueda de nuevas técnicas, menos invasivas y más predecibles en la restauración de la papila interdental, la cual es una realidad que se presenta como secuela en pacientes con periodontopatías. Diversos estudios avalan la efectividad de remodelación y relleno de papila con ácido hialurónico, pero la falta de uniformidad de protocolos empleados y la amplitud de criterios de inclusión hacen confusa la extrapolación de datos clínicos. Este tratamiento mejora los parámetros estéticos estudiados y surge como una alternativa menos invasiva y más predecible.

\section{FUENTE DE FINANCIAMIENTO.}

Este trabajo no cuenta con financiamiento alguno.

\section{RESPONSABILIDADES ÉTICAS}

Los autores declaran que para esta investigación se utilizó el acuerdo con la Asociación Médica Mundial y la Declaración de Helsinki (versión 2008).

\section{CONFIDENCIALIDAD DE DATOS.}

Los autores declaran que en este artículo no aparecen datos de pacientes.

\section{DERECHO A LA PRIVACIDAD Y CONSENTIMIENTO INFORMADO.}

Los autores declaran que en este artículo no aparecen datos de pacientes.

\section{CONFLICTO DE INTERÉS.}

Los autores declaran no tener conflicto de interés.

\section{BIBLIOGRAFÍA}

1. Norndland WP, Tarnow DP. A classification system for loss of papillary height. $J$ Periodontol. 1998 Oct;69(10):1124-1126.

2. Barroeta E, Zabalegui I. Tratamiento estético de la sonrisa en paciente periodonta avanzado: resultados a plazo. A propósito de un caso. SEPA. 2015;1(3): 38-49.

3. Campos-Suaréz M, Peña-Soto C. Ausencia de papila interdental: etiología clasificación y terapéutica. JPAPO. 2016; 1:1-7

4. Lee WP, Kim HJ, Yu SJ, Kim BO. Six month clinical evaluation of interdental papilla reconstruction with injectable hyaluronic acid gel using an image analysis system. Esthet Restor Dent. 2016 Jul;28(4):221-230.

5. Sadat S, Ghasemi M, Salmani Z, Shams N. Clinical application of hyaluronic acid gel for reconstruction of interdental papilla at the esthetic zone. J Islam Dent Assoc Iran. 2013;25(2):152-157

6. Ştirbu I, Popescu S, Tuculina M, Mercuţ V, Manolea H, Iorgulescu D. Hyaluronic acid - innovative therapeutic principle. Key Eng Mater. 2016;695: 41-45.

7. Rohrich RJ, Ghavami A, Crosby MA. The role of hyaluronic acid fillers (Restylane) in facial cosmetic surgery: review and technical considerations. Plast Reconstr Surg 2007;120(Suppl 6):41S-54S.
8. McGuire MK, Scheyer ET. A randomized, double-blind, placebo-controlled study to determine the safety and efficacy of cultured and expanded autologous.fibroblast injections for the treatment of interdental papillary insufficiency associated with the papilla priming procedure. J Periodontol 2007; 78:4-17.

9. Shapiro A. Regeneration of interdental papilla using periodic curettage. Int J Periodont Rest Dent. 1985;5:27-33.

10. Beagle JR. Surgical reconstruction of the interdental papilla. Int J Periodont Rest Dent. 1992;12:145-152.

11. Evian $\mathrm{C}$, Corn $\mathrm{H}$, Rosenberg $\mathrm{E}$. Retained interdental procedure for maintaining anterior esthetics. Compend Contin Educ Dent. 1985 Jan; 6(1):58-64

12. Han TJ, Takei HH. Progress in gingival papilla reconstruction. Periodontol 2000 1996 Jun; $1: 65-8$

13. Becker W, Gabitow I, Stepanov M, Kois J, Smith A, Becker B. Minimally invasive treatment for papillae deficiencies in the esthetic zone: A pilot study. Clin Implant Dent Relat Res. 2010 Mar;12(1):1-8.

14. Chen J, Abatangelo G. Functions of hyaluronan in wound repair. Wound Repair Regen. 1999 Mar-Apr;7(2):79-89. 